\title{
Osteometría-escapulometría glenohumeral en las inestabilidades anteriores recidivantes de hombro. Estudio etiopatogénico de uno de los estabilizadores estáticos mediante tomografía computarizada
}

\author{
Glenohumeral osteometry-scapulometry in anterior shoulder \\ instabilities. Study of one of the static stabilizers through \\ computer tomography
}

\section{S. García-Mata ${ }^{1}$, A. Hidalgo Ovejero ${ }^{1}$}

\section{RESUMEN}

Se realizó un estudio escapulométrico de 98 hombros, mediante TAC: 36 con luxación anterior recidivante (LRH), 37 hombros estables contralaterales de esos pacientes (HEC) y 25 hombros normales (HN). Se evaluaron seis parámetros: índice glenohumeral horizontal, índice glenohumeral vertical, inclinación glenoidea, ángulo de anteversión de la escápula, ángulo glenoideo y retroversión humeral.

Encontramos diferencias estadísticamente significativas entre los grupos LRH y HEC en relación al índice glenohumeral horizontal. Ambos grupos (LRH y HEC) mostraban diferencias significativas en comparación con el grupo de hombros normales (HN) en relación al índice glenohumeral horizontal, inclinación glenoidea y ángulo de anteversión de la escápula. La desproporción cefalo-glenoidea y la inclinación anterior glenoidea son los factores anatómicos desestabilizantes.

Nuestros resultados confirman que, aunque la etiología de la inestabilidad anterior de hombro es multifactorial, existe una predisposición congénita anatómica que la favorece y, en menor grado, también en el hombro estable contralateral, confirmando la existencia de una sutil displasia congénita. Además se revelan como parámetros relevantes en el estudio de la inestabilidad anterior: el índice glenohumeral horizontal, inclinación glenoidea y ángulo de anteversión de la escápula, siendo discutido el papel de la retroversión humeral.

Palabras clave. Luxación anterior recidivante. Hombro. Osteometría. Escapulometrí. Displasia glenohumeral. Predisposición congénita.

\begin{abstract}
We have carried out a scapulometric study, using CT-scan, of 98 shoulders: 36 with recurrent anterior shoulder dislocation (RAD), 37 stable contralateral shoulders (CSS) and 25 normal shoulders (NS). Six parameters were evaluated: Horizontal and Vertical glenohumeral index, glenoid tilt, anteversion angle of the scapula, glenoid angle and humeral retroversion. We found statistically significant differences between the RAD and CSS groups in the horizontal glenohumeral index. Both the RAD and CSS groups showed significant differences in comparison with the NS group in the horizontal glenohumeral index, glenoid tilt and anteversion angle of the scapula. An imbalance of the head-glenoid size and the anterior glenoid tilt are the anatomical factors which favour instability. The determination of these three parameters has great value when assessing patients with anterior shoulder instability.
\end{abstract}

Our results confirm that although the aetiology of anterior glenohumeral instability is multifactorial, there is an anatomical congenital predisposition which favours instability and this predisposition affects (to a lesser extent) the stable contralateral side, confirming the role of subtle congenital dysplasic theory. In addition the following were revealed as relevant parameters in the study of anterior instability: the horizontal glenohumeral index, glenoid tilt and angle of anteversion of the scapula; while the value of the humeral retroversion is under discussion.

Key words. Recurrent anterior dislocation. Shoulder Osteometry. Scapulometry. Glenohumeral dysplasia. Congenital predisposition.
1. Servicio de Cirugía Ortopédica y Traumatología B.

Complejo Hospitalario de Navarra. Pamplona.

Recepción: 30 de enero de 2011

Aceptación provisional: 14 de abril de 2011

Aceptación definitiva: 12 de mayo de 2011

\section{Correspondencia}

Serafín García-Mata

Servicio de Cirugía Ortopédica y Traumatología B Complejo Hospitalario de Navarra

31008 Pamplona. España

e-mail: sgarcima@cfnavarra.es 


\section{INTRODUCCIÓN}

Los primeros estudios anatómicos, de desarrollo y antropométricos del hombro en animales y humanos fueron publicados por Evans en 1945 y Corruccini en 1976 y 1978. Gallino y $\mathrm{col}^{1}$, en un estudio antropométrico, encontraron que la orientación de la fosa glenoidea proporciona una base ósea para la estabilidad de la articulación escapulohumeral tanto en dirección anteroposterior como vertical. El reborde glenoideo proviene de dos centros de osificación que se desarrollan entre los 9-16 años de vida. En la hipoplasia glenoidea ocurre un fallo en el desarrollo del precartílago de la apófisis inferior. Esos pacientes pueden estar asintomáticos o referir dolor y/o inestabilidad $^{2}$. En base a los trabajos de Das y $\mathrm{col}^{3}$ y Saha ${ }^{4,5}$, que observaron que la cavidad glenoidea puede estar en anteversión o en retroversión en relación al plano escapular, se inició la especulación sobre la teoría displásica como factor etiopatogénico primario en la inestabilidad glenohumeral anterior.

En el momento actual está demostrado que la estabilidad del hombro no depende sólo de un único factor como el labrum glenoidalis, cápsula articular ${ }^{6}$, músculo subescapular ${ }^{7}$, ligamentos glenohumerales ${ }^{2,8}$, actividad muscular del hombro, ligamento coracohumeral, fuerza del manguito rotador, de tríceps y porción larga del biceps ${ }^{9}$. Todos ellos tienen un papel, pero en diferentes posiciones del hombro ${ }^{5,6}$. Por tanto, parece obvio que la luxación anterior recidivante de hombro (LRH) no dependa de una sola causa sino más bien sea el resultado de múltiples aspectos etiológicos que le afectan en mayor o menor medida, siendo considerada multifactorial. Wilk y $\mathrm{col}^{9}$ afirmaba que el síndrome clínico de inestabilidad del hombro representa un amplio espectro de síntomas y signos provocados por varios niveles de disfunción. La estabilidad funcional glenohumeral requiere una interacción entre las estructuras estabilizadoras activas y pasivas. Los estabilizadores pasivos incluyen las estructuras capsuloligamentosas, el labrum glenoideo y la geometría o arquitectura ósea.
Una de las hipótesis etiopatogénicas valoradas en la bibliografía es la referente al papel de la displasia glenohumeral, existiendo escasos estudios que hagan referencia a este aspecto. El índice glenohumeral horizontal o transverso es el dato escapulométrico más ampliamente aceptado. Gallie $^{10}$, Merle D'Aubigné y Tubiana ${ }^{11}$ y Trillat y Leclerc-Chalvet ${ }^{12}$ describieron una particular morfología glenoidea con aplanamiento y menor tamaño de lo normal, pero $\mathrm{Saha}^{4}$ fue el primero en conceder importancia a esos parámetros morfológicos óseos como son los índices glenohumerales (vertical y horizontal) y la retrotorsión humeral, realizados mediante radiología. Apenas existen publicaciones que hagan referencia al valor de las alteraciones morfológicas en relación a las inestabilidades glenohumera$\operatorname{les}^{13}$. En los últimos tiempos se han publicado estudios por TAC sobre deformidades secundarias a parálisis braquial obstétrica o prótesis de hombro.

Existen estudios radiológicos goniométricos previos que han confirmado los resultados descritos ${ }^{14-16}$. Es conocido que la posición de la cavidad glenoidea solo puede ser determinada adecuadamente mediante la $\mathrm{TAC}^{17}$. Por esa razón, la goniometría u osteometría glenohumeral no puede ser estandarizada hasta su determinación mediante la $\mathrm{TAC}^{18-22}$. Para realizar este estudio hemos basado nuestras determinaciones en la interpretación triangular de la escápula por TAC descrita por Saha ${ }^{15}$ y Baratelli y Parrini ${ }^{18}$.

El objetivo del presente trabajo es evaluar la morfología ósea glenohumeral (realizando la goniometría mediante TAC) en hombros inestables, hombros contralaterales estables y de personas normales (sin inestabilidades de hombro), especulando sobre la base de una predisposición congénita en la luxación anterior recidivante glenohumeral. Intentamos determinar y analizar unos parámetros descritos (osteometría) para confirmar o negar los estudios previos de Saha (índice glenohumeral vertical) y Baratelli y Parrini ${ }^{18}$ (ángulo de anteversión de la escápula y ángulo glenoideo), que son básicos en el estudio de la etiopatogenia de la inestabilidad anterior de hombro. 


\section{MATERIAL Y MÉTODOS}

Los parámetros evaluados ha sido: índice horizontal glenohumeral, índice vertical glenohumeral, retroversion humeral., inclinación glenoidea, ángulo de anteversión de la escápula y ángulo glenoideo

Los tres últimos parámetros están basados en el concepto de Saha o concepción triangular de la escápula ${ }^{15}$ y en los trabajos de Laumann y Kramps ${ }^{21}$, Bernageau ${ }^{23}$ y Baratelli ${ }^{18}$, que proceden de una construcción geométrica obtenida de Mukherjee y Sivaya, adaptada a la TAC por Lauman ${ }^{21}$. La inclinación glenoidea, ángulo glenoideo y ángulo de anteversión de la escápula forman un triángulo que forma la concepción triangular de la escápula (Fig. 1).

El índice glenohumeral horizontal se define como el producto de la relación entre el diámetro máximo de la glenoides y el de la cabeza humeral, en el plano horizontal (Fig. 2, izquierda). El índice glenohumeral vertical es la misma relación en el plano frontal o coronal (Fig. 2, centro). La retroversion humeral se obtiene de restar el ángulo formado por el eje de la cabeza humeral (eje del cuello humeral) y el eje del capitelum en codo (línea formada que une epicóndilo medial y lateral) ${ }^{19,21,24}$ (Fig. 2, derecha). La inclinación glenoidea es el ángulo formado por el eje glenoideo (la línea que une ambos bordes de la glenoides) y el plano del cuerpo de la escápula (Fig. 3, izquierda). El ángulo de anteversión de la escápula está formado por la línea que pasa a través de ambos rebordes glenoideos en relación con la horizontal (línea base) (Fig. 3 , centro). El ángulo glenoideo se determina por la línea eje de la escápula, en relación con la horizontal o línea base (Fig. 3, derecha).

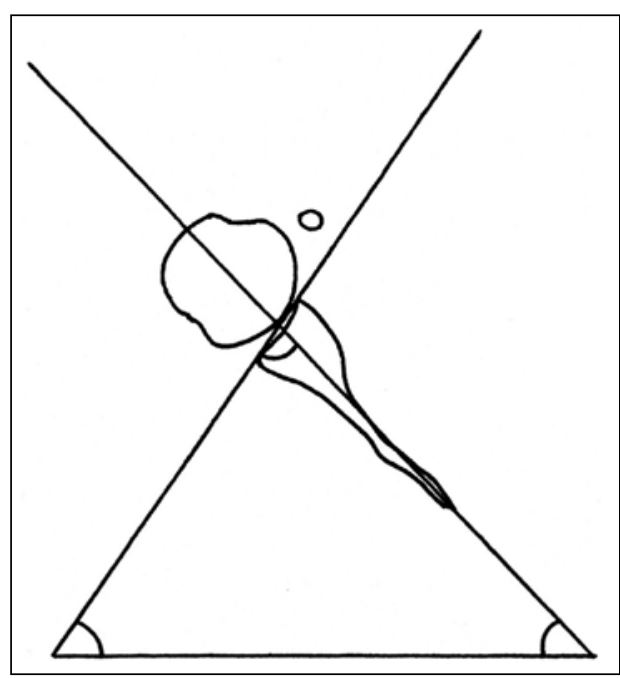

Figura 1. Esquema de la concepción triangular de la escápula (izquierda). Esquema representativo de la determinación del ángulo de retroversión humeral (derecha).

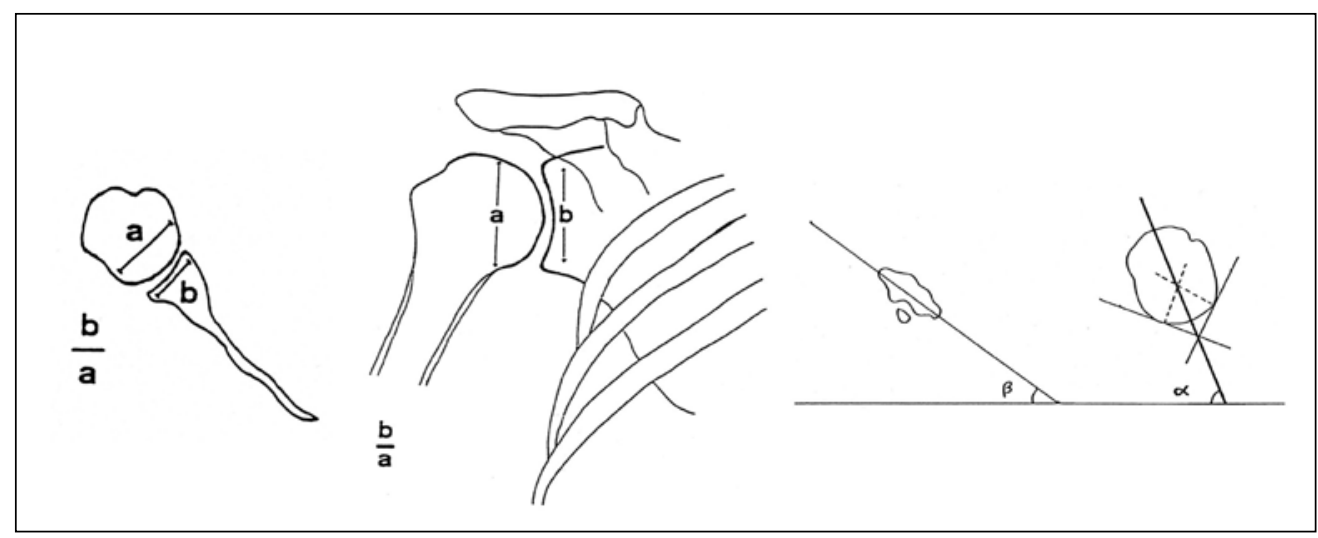

Figura 2. Esquemas de la determinación del Índice glenohumeral horizontal, con corte transversal (izquierda) y del Índice glenohumeral vertical, en corte coronal (derecha). 


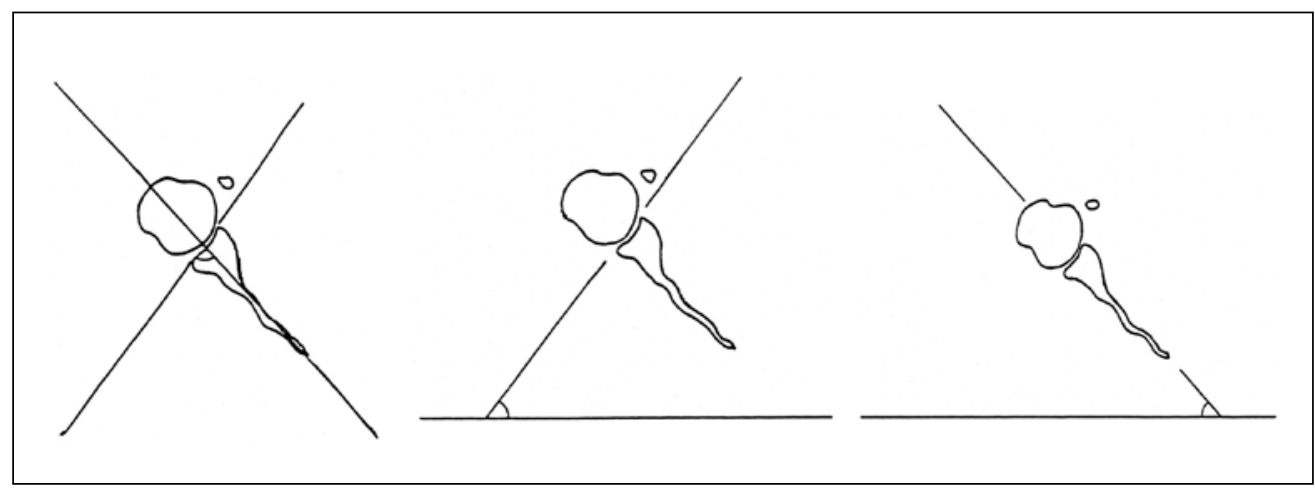

Figura 3. Esquema de la determinación del ángulo de inclinación glenoidea (izquierda), ángulo de anteversión de la escápula (centro) y ángulo glenoideo (derecha) en TAC.

\section{Muestra}

Se evaluaron 36 hombros con luxación anterior recidivante unilateral (grupo LRH) que fueron considerados como válidos (un paciente fue excluido del estudio por degeneración artrósica), 37 hombros estables contralaterales correspondientes a los pacientes del grupo LRH (grupo HEC) y 25 hombros normales de personas sanas no afectas por ninguna patología músculo esquelética (cuyos TAC fueron realizados por otros trastornos regionales) (grupo control de personas asintomáticas o GCPA), evaluándose en total 98 hombros.

\section{Criterios de recidiva y criterios de exclusión}

Se consideró luxación recidivante cuando la luxación había sucedido en, al menos, tres ocasiones según el criterio referido por Collins en 1986.

Fueron criterio de exclusión: historia previa de fractura de escápula, glenoides o húmero, por la posibilidad de presentar una deformidad residual en la estructura ósea; epilepsia; enfermedades del colágeno; hiperelasticidad articular generalizada, según los criterios de Cofield (1987); cirugía previa en la cintura escapular; parálisis braquial obstétrica; enfermedades neurológicas, musculares o neuromusculares; enfermedades reumáticas; lesiones vasculares o nerviosas en la extremidad; historia de sepsis y/o artritis séptica (por la posibilidad de alteración en la estructura ósea que pudiera ocurrir durante su desarrollo); artrosis glenohumeral (pinzamiento articular, esclerosis subcondral y/o osteofitosis); existencia de osteofito en tercio de glenoides aislado; luxaciones recidivantes atraumáticas (primer episodio atraumático).

\section{Características de la muestra}

La edad media de los pacientes en el momento del estudio fue de $22,20 \pm 4,18$ años. Ninguno de los pacientes ni controles realizaban trabajo ni actividad deportiva de lanzamientos que pudieran relacionarse con una alteración secundaria en la morfología. La edad en la primera

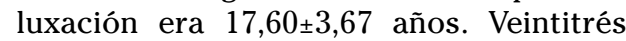
pacientes eran varones $(63,88 \%)$ y $13 \mathrm{mu}-$ jeres $(36,11 \%)$. El lado afecto era derecho en 26 casos $(72,22 \%)$ y en 10 el izquierdo (27,77\%). En 26 casos era el lado dominante (todos ellos en el lado derecho) y en 10 casos era el miembro no dominante (todos los pacientes eran diestros). El número de luxaciones previas era de $10,41 \pm, 17$, con un periodo de evolución de $3,85_{ \pm}, 46$ años. Existía historia familiar positiva en 8 casos 
(22,22\%). El mecanismo de luxación, según Rowe: extensión forzada o abducción en 6 casos (16,66\%), elevación forzada y rotación externa en 19 (52,77\%), contusión directa en el hombro en 9 casos (25\%), caída sobre la mano con el brazo extendido en 2 casos (5,55\%). Con la TAC, como mejor método para el diagnóstico de las lesiones patogénicas óseas glenohumerales ${ }^{13,25-30}$, se evaluaron: lesión de Hill-Sachs o muesca posterior-lateral de la cabeza humeral, que se clasificó en tres tipos según Baratelli y Parrini ${ }^{18}$, con 19 casos como defecto bien definido (52,77\%), 16 como defecto medio $(44,44 \%)$ y uno sin defecto evidente $(2,77 \%)$. La lesión de Bankart podía observarse en 24 casos $(66,66 \%)$ y estaba ausente en $12(33,33 \%)$, en los cuales el despegamiento cápsulo-perióstico, ALPSA o lesión de Broca-Hartmann fue observado en 12 de los 24 casos. En total, las lesiones de Bankart y Broca-Hartmann totalizaban 36 casos (100\%).

El grupo control de personas asintomáticas o GCPA estaba formado por 13 personas (se excluyó un hombro) con una edad media de 26,4 $\pm 5,14$ años, 8 varones y 5 mujeres. En 15 casos se trataba del hombro derecho y diez el izquierdo.

\section{Tomografía axial computarizada}

Los pacientes se colocaban en decúbito dorsal con los antebrazos sobre el abdomen (en ligera rotación interna), posición aceptada para su valoración ${ }^{31}$. Se realizaron cortes transversales cada $5 \mathrm{~mm}$, escogiendo secciones altas para visualizar la lesión de Hill-Sachs, y en el tercio inferior para determinar la existencia de lesión de Bankart y Broca-Hartmann. La determinación goniométrica se realizó en la unión de los tercios inferior y medio de la glenoides, por donde la luxación ocurre ${ }^{24}$, evitando la zona de posible lesión ósea en reborde anterior glenoideo. Debido a que las luxaciones repetidas pueden originar erosión ósea (lesión Bankart) y ello podría variar la determinación del índice glenohumeral horizontal, inclinación glenoidea y ángulo de anteversión de la escápula, el corte era valorado en el nivel inmediato superior si existía lesión de Bankart. El ángulo de retroversion humeral se determinó restando el eje cefálico del húmero y el eje de la paleta humeral en el codo (Fig. 2 , derecha).

Para evitar el sesgo o error interobservador todos los TAC y la determinación de los índices y ángulos fueron evaluados de forma independiente por cada uno de los dos autores. Para evitar sesgo o error intraobservador ambos autores realizaron tres mediciones consecutivas en cada caso. En caso de discrepancia se realizó una nueva revisión por ambos especialistas. La diferencia entre las dos determinaciones independientes en ningún caso excedió un $5 \%$ en cada parámetro.

\section{Procesado y análisis de los datos}

Los análisis se realizaron mediante el programa estadístico SPSS 9.0 for Windows (SPSS Inc., Chicago, Illinois, USA) con las variables estudiadas, test de comparación de medias y test de Kolmogorov-Smirnov para confirmar la distribución normal de la muestra estudiada. Posteriormente, los parámetros descritos o variables cuantitativas entre los diferentes grupos se evaluaron mediante el test de comparación de medias (t-test), considerando el valor de $\mathrm{p} \leq 0,05$ estadísticamente significativo. La comparación entre los grupos se hizo: LRH / HEC; LRH / GCPA; HEC / GCPA.

Se determinó el tamaño de la muestra, encontrando entre 25 y 34 sujetos por grupo para un $\alpha=0,05$ unilateral y un $\beta=0,20$ y un valor de efecto esperado entre 0,70 y 0,60 , que es el efecto encontrado en la variable principal de este trabajo.

En cada una de las submuestras de los grupos descritos (LRH, HEC, GCPA) los seis parámetros fueron estudiados en relación al sexo, edad, lado y dominancia por medio del t-test. Se empleó el índice de correlación para comparar los parámetros con la edad de la primera luxación y con la edad de exploración. 


\section{RESULTADOS}

\section{A. Comparación de los tres grupos (LRH, HEC, GCPA)}

A.1. En la comparación entre el grupo de pacientes con luxación anterior recidivante (LRH) y el grupo de hombros estables contralaterales de esos pacientes (HEC), se hallaron diferencias estadísticamente significativas en el índice gle- nohumeral horizontal, siendo menor el valor de dicho índice en los hombros con luxación recidivante $(0,62 \pm 0,05)$ respecto del hombro sano contralateral $(0,68 \pm$ $0,06)$. Los resultados encontrados con el resto de los parámetros eran muy similares (Tabla 1). Por tanto la desproporción céfalo-glenoidea parece ser el factor predisponente anatómico fundamental del lado afecto, o inestable, respecto al sano contralateral.

Tabla 1. Comparación de los parámetros entre el grupo de hombros con LRH y el grupo de hombros HEC

\begin{tabular}{l|c|c|c}
\hline & LRH & HEC & $\begin{array}{c}\text { Significación } \\
\text { estadística }\end{array}$ \\
\hline Índice glenohumeral horizontal & $\begin{array}{c}0,62 \pm 0,05 \\
(\mathrm{DE}=0,009)\end{array}$ & $\begin{array}{c}0,68 \pm 0,065 \\
(\mathrm{DE}=0.011)\end{array}$ & $\mathrm{P}=0,000$ \\
Inclinación glenoidea & $\begin{array}{c}85,25^{\circ} \pm 2,94^{\circ} \\
(\mathrm{DE}=0,49)\end{array}$ & $\begin{array}{c}84,45^{\circ} \pm 3,86^{\circ} \\
(\mathrm{DE}=0,63)\end{array}$ & $\mathrm{P}=0,330$ \\
& $\begin{array}{c}44,24^{\circ} \pm 4,15^{\circ} \\
(\mathrm{DE}=0,70)\end{array}$ & $\begin{array}{c}44,71^{\circ} \pm 3,98^{\circ} \\
(\mathrm{DE}=0,65)\end{array}$ & $\mathrm{P}=0,626$ \\
Ángulo de anteversión de la escápula & $50,46^{\circ} \pm 3,75^{\circ}$ & $50,82^{\circ} \pm 4,14^{\circ}$ & \\
Ángulo glenoideo & $(\mathrm{DE}=0,63)$ & $(\mathrm{DE}=0,68)$ & $\mathrm{P}=0,704$ \\
& $19,55^{\circ} \pm 12,46^{\circ}$ & $19,58^{\circ} \pm 12,06^{\circ}$ & \\
Retroversión humeral & $(\mathrm{DE}=3,02)$ & $(\mathrm{DE}=2,92)$ & $\mathrm{P}=0,994$ \\
& $0,95 \pm 0,10$ & $0,93 \pm 0,11$ & $\mathrm{P}=0,466$ \\
Índice glenohumeral vertical & $(\mathrm{DE}=0,02)$ & $(\mathrm{DE}=0,02)$ & \\
\hline
\end{tabular}

A.2. Al comparar el grupo de hombros con luxación anterior recidivante (LRH) y el grupo de hombros normales pertenecientes a la población normal (GCPA) se encontraron diferencias estadísticamente significativas $(\mathrm{p}<0,001)$ en los siguientes tres parámetros:

a) Índice glenohumeral horizontal: siendo su valor menor en los hombros afectos de luxación recidivante $(0,62$ $\pm 0,005$ ) que en el grupo de hombros normales $(0,76 \pm 0,01)$.

b) Inclinación glenoidea: con un valor mayor en el grupo de luxación recidi- vante $\left(85,25^{\circ} \pm 2,94^{\circ}\right)$ que en el grupo control de personas asintomáticas $\left(78,96^{\circ}\right)$.

c) Ángulo de anteversión de la escápula: con un valor menor en el grupo de luxación recidivante $\left(44,24^{\circ} \pm 4,15^{\circ}\right)$ que en el grupo control de personas asintomáticas $\left(49,08^{\circ} \pm 1,64^{\circ}\right)$.

La diferencia en el valor del ángulo glenoideo no era significativa. La retroversion humeral y el índice glenohumeral vertical no pudieron ser comparadas ya que el grupo control de personas asintomáticas (GCPA) no pudo ser evaluado (Tabla 2). 
Tabla 2. Comparación de los parámetros entre el grupo de hombros LRH y el grupo GCPA

\begin{tabular}{|c|c|c|c|}
\hline & LRH & GCPA & $\begin{array}{c}\text { Significación } \\
\text { estadística }\end{array}$ \\
\hline Índice glenohumeral horizontal & $\begin{array}{c}0,62 \pm 0,05 \\
(\mathrm{DE}=0,009)\end{array}$ & $\begin{array}{c}0,76 \pm 0,010 \\
(\mathrm{DE}=0,02)\end{array}$ & $P=0,000$ \\
\hline Inclinación glenoidea & $\begin{array}{c}85,25^{\circ} \pm 2,94^{\circ} \\
(\mathrm{DE}=0,49)\end{array}$ & $\begin{array}{c}78,96^{\circ} \pm 2,80^{\circ} \\
(\mathrm{DE}=0,56)\end{array}$ & $P=0,000$ \\
\hline Ángulo de anteversión de la escápula & $\begin{array}{c}44,24^{\circ} \pm 4,15^{\mathrm{o}} \\
(\mathrm{DE}=0,70)\end{array}$ & $\begin{array}{c}49,08^{\circ} \pm 1,84^{\circ} \\
(\mathrm{DE}=0,36)\end{array}$ & $P=0,000$ \\
\hline Ángulo glenoideo & $\begin{array}{c}50,46^{\circ} \pm 3,75^{\circ} \\
(\mathrm{DE}=0,63)\end{array}$ & $\begin{array}{c}51,96^{\circ} \pm 2,38^{\circ} \\
(\mathrm{DE}=0,47)\end{array}$ & $P=0,086$ \\
\hline Retroversión humeral & $\begin{array}{c}19,55^{\mathrm{o}} \pm 12,46^{\mathrm{o}} \\
(\mathrm{DE}=3,02)\end{array}$ & No muestra & - \\
\hline Índice glenohumeral vertical & $\begin{array}{c}0,95 \pm 0,10 \\
(\mathrm{DE}=0,02)\end{array}$ & No muestra & - \\
\hline
\end{tabular}

A tenor de estos resultados, la diferencia morfológica entre el hombro inestable y el grupo control de personas asintomáticas (población no afecta de inestabilidad glenohumeral) se debe a la desproporción céfalo-glenoidea así como a la inclinación anterior excesiva de la glenoides.

A.3. Al comparar los hombros sanos contralaterales de los pacientes con LRH (HEC) y el grupo control de personas asintomáticas de la población normal (GCPA), se encontraron diferencias estadísticamente significativas $(\mathrm{p}<0,001)$ en los mismos tres parámetros:
a) Índice glenohumeral horizontal: con un valor menor en el grupo HEC $(0,68$ $\pm 0,06)$ que en el GCPA $(0,76 \pm 0,10)$.

b) Inclinación glenoidea: con un valor mayor en el grupo HEC $\left(84,45^{\circ} \pm\right.$ $\left.3,86^{\circ}\right)$ que en el GCPA $\left(78,96^{\circ} \pm 2,8^{\circ}\right)$.

c) Ángulo de anteversión de la escápula: encontramos un valor inferior en el grupo HEC $\left(44,71^{\circ} \pm 3,98^{\circ}\right)$ en comparación con el GCPA $\left(49,08^{\circ} \pm 1,84^{\circ}\right)$. Encontramos el ángulo glenoideo ligeramente mayor en el grupo control de personas asintomáticas (GCPA) sin que la diferencia sea estadísticamente significativa.

Los valores de la retroversion humeral $\mathrm{y}$ del índice glenohumeral vertical no pudieron ser comparados ya que no fueron determinados en el grupo GCPA (Tabla 3).

Tabla 3. Comparación de los parámetros entre el grupo HEC y el grupo GCPA

\begin{tabular}{|c|c|c|c|}
\hline & HEC & GCPA & $\begin{array}{c}\text { Significación } \\
\text { estadística }\end{array}$ \\
\hline Índice glenohumeral horizontal & $\begin{array}{c}0,68 \pm 0,06 \\
(\mathrm{DE}=0,01)\end{array}$ & $\begin{array}{c}0,76 \pm 0,010 \\
(\mathrm{DE}=0,02)\end{array}$ & $P=0,001$ \\
\hline Inclinación glenoidea & $\begin{array}{c}84,45^{\circ} \pm 3,86^{\circ} \\
(\mathrm{DE}=0,63)\end{array}$ & $\begin{array}{c}78,96^{\circ} \pm 2,80^{\circ} \\
(\mathrm{DE}=0,56)\end{array}$ & $P=0,000$ \\
\hline Ángulo de anteversión de la escápula & $\begin{array}{c}44,71^{\circ} \pm 4,98^{\circ} \\
(\mathrm{DE}=0,65)\end{array}$ & $\begin{array}{c}49,08^{\circ} \pm 1,84^{\circ} \\
(\mathrm{DE}=0,36)\end{array}$ & $P=0,000$ \\
\hline Ángulo glenoideo & $\begin{array}{c}50,82^{\mathrm{o}} \pm 4,14^{\mathrm{o}} \\
(\mathrm{DE}=0,68)\end{array}$ & $\begin{array}{c}51,96^{\circ} \pm 2,38^{\circ} \\
(\mathrm{DE}=0,47)\end{array}$ & $P=0,221$ \\
\hline Retroversión humeral & $\begin{array}{c}19,58^{\circ} \pm 12,06^{\circ} \\
(\mathrm{DE}=2,92)\end{array}$ & No muestra & - \\
\hline Índice glenohumeral vertical & $\begin{array}{l}0,93 \pm 0,11 \\
(\mathrm{DE}=0,02)\end{array}$ & No muestra & - \\
\hline
\end{tabular}


Por lo tanto, la diferencia estadísticamente significativa de tres de los parámetros estudiados (índice glenohumeral horizontal, inclinación glenoidea y ángulo de anteversión de la escápula) en la comparación de los grupos LRH y GCPA así como entre los grupos HEC y GCPA muestran que existe una diferencia evidente en la morfología entre los hombros normales y los hombros de los pacientes afectos de LRH (tanto en el hombro inestable como en el estable contralateral). De tal forma, podemos conjeturar que existe un cierto grado de displasia en ambos hombros de los pacientes que presentan una luxación anterior recidivante de hombro unilateral (tanto en el hombro inestable como en el sano contralateral), que supondría un cierto grado de predisposición anatómica o morfológica. La única diferencia significativa entre los hombros de los grupos LRH y HEC confirma la leve diferencia entre los hombros de los pacientes.

En las tablas 2 y 3 no se valoran el índice glenohumeral vertical y la retroversión humeral en el grupo control de personas asintomáticas (GCPA) u hombros normales debido a que no fueron determinados. La explicación es que el Índice glenohumeral vertical precisa de un corte coronal añadido de la articulación y la retroversión humeral exige un corte asociado en el codo (eje bicondíleo) para poder determinarla (Fig. 2 derecha). Ambos tipos de corte no se obtuvieron en el grupo control de personas asintomáticas o GCPA porque los TAC se obtuvieron de pacientes con diferentes procesos (distintos a los traumáticos y ortopédicos). En ellos aprovechamos los cortes que pasaban por tórax y hombro para poder utilizarlos si estaban en la posición adecuada del resto de los grupos. Obviamente no se consideró ético someterlos a otros cortes distintos o en otra localización sin una utilidad para el proceso del paciente ni su conocimiento.

B. Los seis parámetros fueron estudiados en relación a cinco variables: edad de la primera luxación, edad en el momento del estudio, género, lado afecto y dominancia en cada una de las submuestras de los grupos descritos:

B.1. En la submuestras del grupo LRH: no hubo diferencia significativa con respecto a las variables género, lado y dominancia. Por el contrario, sí se encontró diferencia significativa en relación a las variables: edad de la primera luxación $(\mathrm{p}<0.001)$ y edad en el momento de al realización del estudio $(\mathrm{p}<0,001)$ para el índice glenohumeral vertical.

B.2. En la submuestras del grupo HEC: no se encontraron diferencias estadísticamente significativas en relación a ninguna de las cinco variables estudiadas.

B.3. En la submuestras del grupo GCPA: tampoco se encontraron diferencias significativas.

\section{DISCUSIÓN}

Es aceptado que las lesiones óseas de la cabeza humeral (Hill-Sachs) y/o glenoidea (Bankart), junto con lesiones cápsuloligamentosas, son responsables de las recidivas en las luxaciones glenohumerales. Es conocida la relación de un defecto glenoideo (Bankart) con la inestabilidad anteroinferior. Un defecto óseo con una anchura de, al menos, $21 \%$ de la glenoides puede causar inestabilidad $^{32}$. Un defecto glenoideo anterior de $6 \mathrm{~mm}$ (equivale al 19\% de la anchura glenoidea) causa inestabilidad, incluso tras reparación de la lesión de Bankart, si dicho defecto permanece ${ }^{33}$.

La relación entre la displasia glenoidea y la inestabilidad es bien conocida en la evolución de la parálisis braquial obstétrica $(\mathrm{PBO})^{19}$. La retroversión glenoidea media en los hombros luxados como secuela de la PBO disminuye progresivamente desde un $31 \%$ tras la reducción abierta y sigue mejorando de forma continua un 9\% por año. Sin embargo, pocos trabajos publicados se han referido a la relación entre hipoplasia glenoidea e inestabilidad de hombro en la población normal ${ }^{2,20,34-36}$.

El término de displasia glenoidea congénita o primaria se refiere a una rara condición caracterizada por una osificación 
incompleta de los $2 / 3$ inferiores de la glenoides cartilaginosa y el adyacente cuello de la escápula. La displasia es más frecuente de lo que se pensaba, es una condición asintomática y tiene una clara relación con la inestabilidad ( $1 / 3$ desarrollan síntomas de inestabilidad). Embriológicamente Gadner en 1953 lo explicaba al describir dos centros de osificación secundarios en la escápula: base de la apófisis coracoides y en zona medio-glenoidea, en los cuales se desarrolla un aplanamiento o hipoplasia de la glenoides. Neviaser refiere que la hipoplasia congénita es asintomática, pero en ocasiones (al llegar la pubertad) puede observarse inestabilidad glenohumeral. Esto confirma que la displasia congénita es un factor desestabilizante.

Entre ambas condiciones (defectos óseos e hipoplasia congénita) pueden existir ligeras variaciones de la morfología glenoidea y de relación glenohumeral, que representarían un tipo menos evidente de displasia, que pudieran favorecer la tendencia a la inestabilidad, que ha sido objetivo de nuestro estudio.

Matsen y $\operatorname{col}^{37}$ ya explicaban que algunos factores que contribuyen a la inestabilidad glenohumeral están congénitamente determinados: tamaño y profundidad de la glenoides, torsión humeral y la inclinación de la cavidad glenoidea. Baratelli y Parrini encontraron que la glenoides tipo $\mathrm{C}$ de Saha (radio glenoideo menor que el de la cabeza humeral) es la condición más inestable de la articulación glenohumeral, de forma estadísticamente significativa ${ }^{18}$. No compartimos la opinión de Randelli y Gambrioli acerca de que la orientación y dimensión de la cavidad glenoidea varíe solo como resultado de cambios traumáticos o degenerativos ${ }^{24}$, ya que en el presente trabajo la artrosis fue un criterio de exclusión y los cortes de la TAC no se evaluaron en la zona traumática de la glenoides, sino proximalmente a ella. La osteometría o escapulometría la realizamos mediante la determinación de varios parámetros escapulométricos: el índice glenohumeral horizontal (IGGH), el índice glenohumeral vertical y la inclinación glenoidea.

\section{Índice glenohumeral horizontal}

El valor normal según autores, oscila entre $0,57 \pm 0,053$ y $0,68 \pm 0,042$. Hemos encontrado un valor discretamente superior en el grupo HN respecto a lo que la bibliografía refiere. Esas cifras pueden variar ya que trabajos precedentes fueron realizados mediante diferentes proyecciones radiográficas y nosotros por TAC. Es destacable la diferencia significativa entre los diferentes grupos estudiados con el mismo método.

Según nuestros resultados puede observarse que a mayor estabilidad de la articulación corresponde un valor mayor del índice glenohumeral ( $\mathrm{LRH}=0,62, \mathrm{HEC}=0,68$, $\mathrm{GCPA}=0,76$ ), coincidiendo con otros autores $^{4,16,18,38}$. Randelli también encontró diferencia entre los hombros normales y afectos de LRH, aunque no significativa ${ }^{24}$. La diferencia estadísticamente significativa de este índice entre el grupo de afectos de luxación anterior recidivante (LRH) en comparación con los otros dos grupos (HEC, GCPA) así como la existente entre el grupo de hombro sano contralateral (HEC) y los controles (GCPA), revela la existencia de un cierto grado de displasia que afecta de forma bilateral (a pesar de que luxación recidivante sea unilateral), aunque en mayor grado en el lado con inestabilidad clínica. Por tanto, encontramos que existe una base congénita (desproporción céfalo-glenoidea), en el IGHH del hombro inestable y también en el HEC, el cual también presenta diferencia estadísticamente significativa (aunque en menor grado) en relación a los hombros de personas normales (GCPA). Todo ello confirma que un IGHH disminuido es un factor predisponente de inestabilidad anterior: cuanto menor sea la cavidad glenoidea en relación a la cabeza humeral, mayor es la posibilidad de desencadenarse inestabilidad, la cual se desarrollará o no según existan otros factores adicionales o predisponentes. Esto explicaría por qué unos hombros se luxan y otros no.

Esa alteración no se explica simplemente por una lesión traumática del reborde anterior glenoideo, ya que dicha lesión era evitada en la selección de los cortes toma- 
dos en la TAC. Además, el hombro estable contralateral (HEC) no tenía lesión en el reborde glenoideo anterior como podía ocurrir en el hombro inestable y no existían diferencias significativas entre ambos hombros (HEC) y (LRH). Sin embargo, ambos grupos sí mostraban diferencias significativas en relación al grupo de hombros de la población normal de control (GCPA). De todas formas, una lesión traumática grande del reborde anterior glenoideo incrementa el grado de displasia inicial y puede desencadenar la inestabilidad. Ya hemos referido previamente que un defecto óseo de $19-21 \%$ de la glenoides puede causar inestabilidad $^{32,33}$, incluso tras reparación de la lesión de Bankart, si dicho defecto permanece.

\section{Índice glenohumeral vertical}

Índice descrito por $\mathrm{Saha}^{4}$ con radiografías $(0,75 \pm 0,03)$. Posteriormente, Maki y Gruen ${ }^{39}$ referían un valor normal de 0,86 . En nuestro estudio (con TAC) obtuvimos un valor de $0,95 \pm 0,10$ en hombros con $\mathrm{LRH}$, $\mathrm{y}$ de $0,93 \pm 0,11$ en los hombros estables contralaterales (HEC). No encontramos relación entre éste parámetro y la inestabilidad glenohumeral, al igual que Saha ${ }^{4}$.

\section{Inclinación glenoidea}

Das demostró que la mayor parte de los hombros inestables presentan un aumento de la inclinación glenoidea ${ }^{3}$. Saha comparó individuos normales y afectos de LRH, encontrando que la mayor parte de los individuos normales tenían un grado variable de inclinación posterior, mientras que la mayor parte de los hombros inestables mostraban que la inclinación glenoidea era en dirección anterior, concluyendo que la inclinación glenoidea favorece la luxación anterior ${ }^{22}$. Kuriyama y col $^{40}$ encontraron un valor en individuos normales de $82,94^{\circ}$ de inclinación glenoidea, por $98,72^{\circ}$ en hombros con luxación única y $99,27^{\circ}$ en aquellos hombros con LAH. También aquí es posible deducir que a mayor grado de inestabilidad anterior, mayor ángulo de inclinación glenoidea anterior. Es importante recalcar que esos autores realizaron sus estudios por medio de radiología convencional (en diferentes proyecciones). Posteriormente, algunos autores ${ }^{40,41}$ estudiaron esos parámetros por medio de la TAC, describiendo una ligera diferencia en la inclinación glenoidea entre los individuos. Más tarde, Baratelli y Parrini con TAC encontraron un valor de $82,3^{\circ}$ en pacientes con $\mathrm{LRH}$, y $77,4^{\circ}$ en aquellos que habían sufrido un único episodio de luxación en los cinco últimos años, siendo la diferencia entre ambos grupos estadísticamente significativa ${ }^{18}$. A partir de ello puede deducirse que entre aquellas personas que sufren luxación única y aquellas en las que se convierte en recidivante, existe una diferencia morfológica o displásica que predispone o contribuye a la inestabilidad crónica. Según nuestros resultados, similares a los de Baratelli y Parrini, a mayor grado de inestabilidad del hombro mayor es el valor de la inclinación glenoidea anterior: $\mathrm{LAH}=85,25^{\mathrm{o}}$; CSS $=84,45^{\circ} ; \mathrm{GCPA}=78,96^{\circ}$, la diferencia entre el grupo de hombros afectos de LRH y el grupo de hombros estables contralaterales (HEC) no era significativa. Sin embargo, sí existía diferencia significativa entre los grupos de LRH y HEC al compararlos con el grupo de población normal (GCPA), lo cual evidencia que la displasia afecta de forma bilateral, explicándose la mayor frecuencia de inestabilidad, a largo plazo, en el hombro estable contralateral ${ }^{7,8,42}$. El desarrollo de una luxación recidivante dependerá de la alteración de otros parámetros escapulométricos así como del resto de los factores etiopatogénicos que favorecen su mayor frecuencia de aparición en el lado dominante, como resultado de haber estado sometido a una mayor cantidad o intensidad de traumatismos.

Randelli y Gambrioli ${ }^{24}$ opinaban que valores menores en la inclinación glenoidea se debían exclusivamente a la erosión o fractura del reborde anterior glenoideo, y que los valores mayores en dicho parámetro eran debidos a osteofitos dicho reborde. Paradójicamente, encontraba valores similares en el tercio inferior, medio y superior de la glenoides en el grupo de hombros 
normales y en los afectos de LRH. Al deberse a causa traumática la erosión o fractura del reborde glenoideo debiera mostrar valores diferentes en los tercios medio y superior de la glenoides en relación al inferior (es donde se localizan dichas lesiones traumáticas óseas). En nuestro estudio la posibilidad de alteración por osteofitosis no se contemplaba ya que era criterio de exclusión, y en los casos con erosión del reborde glenoideo se realizaba la medida en el corte más proximal sin lesión.

En 2010 Bryce y col ${ }^{43}$ han demostrado que la versión glenoidea tiene un valor relativo y valor dinámico, variando con la rotación sagital y coronal de la escápula. Así, cuando la escápula era rotada en el plano coronal, la versión glenoidea medía $10,6^{\circ}$ de anteversión a $20^{\circ}$ de abducción y $4,6^{\circ}$ a $20^{\circ}$ de adducción. En el plano sagital la rotación interna resulta en relativa anteversión, y la rotación externa en relativa retroversión. Rouleau y $\mathrm{col}^{44}$, en 2010 , encontraron fiable la medición de la versión glenoidea mediante TAC bidimensional.

\section{Ángulo de anteversión de la escápula (AAE)}

Baratelli y Parrini ${ }^{18}$ calcularon el valor de este parámetro en pacientes con LRH $\left(43,8^{\circ}\right)$ y en aquellos que había sufrido una única luxación $\left(46,9^{\circ}\right)$, no encontrando diferencia significativa entre ellos, pero recalcando un valor mayor en el grupo más estable. Mintzer y $\mathrm{col}^{45}$, por TAC y RNM, determinaron la versión glenoidea de 111 hombros normales de niños, encontrando que la glenoides está en mayor retroversión durante los dos primeros años de vida $\left(-6,3^{\circ} \pm 6,5^{\circ}\right)$ y al final de la primera década de la vida alcanza la versión glenoidea adulta $\left(1,7^{\circ} \pm 6,4^{\circ}\right.$ ) (diferencia estadísticamente significativa). Este efecto de retroversion glenoidea explicaría la bajísima tasa de luxaciones en la primera década de la vida.

Schlemmer y col ${ }^{46}$ evaluaron, con TAC, a 30 personas libres de inestabilidad glenohumeral, encontrando que existe una torsión espiral en la superficie articular de la cavidad glenoidea con progresiva disminu- ción en la retroversión glenoidea desde la parte superior a la inferior de la cavidad en el 95\% de los hombros. La versión glenoidea era mayor en el lado dominante.

No hemos encontrado otros trabajos que hagan referencia a este parámetro en hombros normales. En el grupo de hombros normales (GCPA) obtuvimos un valor de $49,08^{\circ} \pm 1,84^{\circ}$, mayor $(\mathrm{p}<0,001)$ que en el grupo de hombros afectos LRH $\left(44,24^{\circ}{ }_{ \pm}\right.$ $4,15^{\circ}$ ) y también mayor que el grupo de HEC de los pacientes con LRH (valor medio de $\left.44,71^{\circ} \pm 3,98^{\circ}\right)$. Ello indica que a mayor ángulo de anteversión de la escápula, mayor estabilidad: $\mathrm{GCPA}=49,08^{\circ}$, $\mathrm{HEC}=44,71^{\circ}$, $\mathrm{LRH}=44,24^{\circ}$. También respecto a éste parámetro puede afirmarse que existe un factor morfológico o displásico que diferencia los hombros inestables (LRH) y los hombros estables contralaterales de esos pacientes (HEC) respecto de los hombros estables de la población normal (GCPA), pero no entre los dos primeros grupos (LRH / HEC).

La contribución en la estabilidad glenohumeral del AAE es incierta ${ }^{18}$. Barton ${ }^{47}$ encontró pocos casos de AAE anormal en los que observaron agenesia de trapecio, clavícula corta y deformidad torácica como explicación probable de la excesiva anteversión (displásicos). Baratelli y Parrini conjeturaban que el AAE está relacionado con el equilibrio de la actividad muscular axialescapular, de tal forma que dicho ángulo representa un ajuste funcional para mejorar la estabilidad de un hombro con equilibrio muscular alterado ${ }^{18}$. Según recientes estudios de Bryce y col $^{43}$ sabemos que cualquier defecto de alineación $\geq 1^{\circ}$ de la escápula en los planos sagital o coronal creará imprecisiones en la medición de la versión glenoidea. El plano de reconstrucción axial debe ser alineado con la escápula cuando las imágenes de la TAC bidimensional son usadas para medir la versión glenoidea, por lo que sus hallazgos apoyan el uso de modelos tridimensionales para evaluar dicha versión. $\mathrm{Al}$ evaluar la versión glenoidea por la TAC, el plano de reconstrucción axial debe tenerse en cuenta. En cambio, la versión glenoidea medida sobre modelos tridimensionales es independiente de la posición escapular ${ }^{43}$. 
Por lo tanto, pensamos que la movilidad de la escápula evaluada por su ángulo de anteversión puede ser un factor estabilizador de importancia en la articulación glenohumeral. La disminución de dicho ángulo (y, por tanto, mayor ángulo glenoideo), con el mismo grado de rotación externa, puede causar mayor tensión en el mecanismo capsular anterior, provocando un efecto similar al incremento de la rotación externa, así como una predisposición a la degeneración temprana de dichas estructuras por el estrés repetido que desencadenaría el mismo fenómeno patogénico descrito sobre la inclinación glenoidea.

\section{Ángulo glenoideo}

Saha ${ }^{4}$ describió cómo el ángulo glenoideo se afecta por la inclinación glenoidea anatómica superior de la glenoides y por el ángulo escapulotorácico. No hemos encontrado una determinación de este ángulo en la población normal. En el trabajo obtuvimos un valor medio de $51,96^{\circ}$ $\pm 2,38^{\circ}$. Ozaki ${ }^{31}$, con cinerradiografia, confirmó que cuando dicho valor disminuye la estabilidad glenohumeral aumenta. En hombros con inestabilidad involuntaria inferior y mutidireccional encontró que el ángulo glenoideo disminuye ligeramente con la abducción, alcanzando un valor mínimo a $180^{\circ}$ de abducción $\left(52,2^{\circ} \pm 23,2^{\circ}\right)$, concluyendo que en el plano escapular el valor del ángulo glenoideo es mayor en los hombros patológicos que en los normales. Baratelli y Parrini ${ }^{18}$ encontraron valores de $54^{\circ}$ para hombros con de LRH, y $55,9^{\circ}$ en los que habían sufrido una luxación única en los cinco años precedentes, pero sin diferencia estadísticamente significativa. Ello fue interpretado como el resultado de una disminución "funcional» del ángulo de anteversión en hombros con una elevada inclinación glenoidea. Demostraba que el ángulo glenoideo aumenta o disminuye dependiendo de si la inclinación glenoidea hace lo mismo y varía inversamente con el aumento o disminución del ángulo de anteversión de la escápula. Los resultados de nuestro estudio confirman los estudios precedentes por medio de comparación de grupos bien diferenciados: $\mathrm{LRH}=50,46^{\circ}$, $\mathrm{HEC}=50,82^{\circ}, \mathrm{GCPA}=51,96^{\circ}$. La ausencia de diferencias significativas entre ellos parece reafirmar el valor insignificante de este parámetro, lo cual es lógico ya que su valor está en relación con otros dos parámetros con diferencias estadísticamente significativas (inclinación glenoidea y ángulo de anteversión de la escápula). Al realizar la TAC el ángulo glenoideo es firme ya que el paciente está en una posición estática. Pero su adaptación dinámica a los movimientos de la articulación del hombro podría compensar alteraciones de otros parámetros (inclinación glenoidea y ángulo de anteversión de la escápula).

\section{Retroversión humeral (RH)}

Los estudios antropométricos realizados ofrecen un valor medio de $\mathrm{RH}$ de $30^{\circ}$, considerando valores normales $20^{\circ}-40^{\circ}$ $23,26,31,42$. Saha encontró un valor radiológico de $30^{\circ}\left(40^{\circ}-60^{\circ}\right)$ en la población hindú normal y $32^{\circ}-48^{\circ}$ en pacientes afectos de LRH. Encontró que la mayor retrotorsión humeral en primates es compensada por la fuerza de los músculos estabilizadores horizontales y un IGHH aumentado ${ }^{4}$. Debevoise y $\operatorname{col}^{48}$ y Dunlap y $\operatorname{col}^{49}$ introdujeron el cálculo de los ángulos corregidos, estableciendo un ángulo de torsión humeral normal de $61,2^{\circ}\left(47^{\circ}-85^{\circ}\right)$ y de $76,5^{\circ}\left(63^{\circ}-104^{\circ}\right)$ en pacientes afectos de LRH. Se ha demostrado una alta correlación entre $\mathrm{Rx}$ y TAC en la determinación de la RH, no encontrando diferencias entre sexos. Cyprien y col${ }^{38}$, con proyección axilar modificada, evaluó la $\mathrm{RH}$ proyectada y la modificada en hombros normales y en inestables con LRH concluyendo que la $\mathrm{RH}$ no parece desempeñar un papel en pacientes con LRH, posteriormente confirmado por Vasey y $\mathrm{col}^{16}$. Bernageau y Godefroy, con TAC como método más sencillo y exacto, encontraron un valor normal de $25^{\circ}-40^{\circ}{ }^{23}$. La RH por medio de la TAC realizada por Randelli y Gambrioli ${ }^{24}$, en hombros normales $\left(25^{\circ}-35^{\circ}\right)$ y con LRH $\left(28^{\circ}-35^{\circ}\right)$ revelaba ausencia de diferencias significativas. Lauman y Kramps ${ }^{21}$, también con TAC, refieren $28^{\circ}-31^{\circ}$ en pacientes con LRH (valores similares a los casos norma- 
les). Por tanto, el valor de la RH en relación a la LRH es controvertido.

Kronberg, en 1990, con radiología convencional encontró diferencias significativas entre el lado dominante $\left(30^{\circ}-35^{\circ}\right)$ y el no dominante $\left(26^{\circ}-31^{\circ}\right)$, sin diferencias por sexo, atribuyéndolo a variaciones en el desarrollo durante la infancia y dependiendo del uso realizado de cada extremidad. También demostró que a mayor $\mathrm{RH}$, mayor rango de rotación externa, así como un menor grado de $\mathrm{RH}$ en luxaciones atraumáticas respecto de las traumáticas, y en los hombros inestables respecto de los estables ${ }^{50}$. Hernigou y coll $^{51}$, con TAC en húmeros de cadáveres, refería que el ángulo variaba poco $\left(2,1^{\circ}\right)$ entre los dos húmeros normales del mismo individuo. Schlemmer y $\mathrm{col}^{46}$ concluyen que la mayor RH se correlaciona con una versión glenoidea menos pronunciada y viceversa.

Nuestro estudio revela un valor de $19,55^{\circ}$ de $\mathrm{RH}$ en los hombros afectos de LRH, con una gran dispersión de valores (desviación estándar 12,46º ). La ausencia de diferencias significativas con otros grupos de estudio nos confirma la opinión generalizada de que la RH no parece tener una incidencia significativa en la LRH.

Una limitación de este estudio es la ausencia de diferenciación entre los hombros denominados TUBS (Traumatic, Unilateral, Bankart lesion, Surgery) y AMBRII (Atraumatic, Multidirectional, Bilateral, Rehabilitation, Involuntary, Inferior capsular shift) (en el grupo LRH). Podemos especular sobre la posibilidad de un mayor grado de predisposición o de displasia glenohumeral en los hombros tipo AMBRII. La etiología traumática del primer episodio en todos los casos del grupo LRH nos inducen a pensar que sean tipo TUBS los hombros evaluados. Inui y col${ }^{34}$ estudiaron por RNM inestabilidades con dirección posterior, encontrando la versión glenoidea alterada de forma estadísticamente significativa. La pérdida de la inclinación glenoidea y concavidad de la glenoides inferior se correlaciona con la dirección de la traslación en la inestabilidad posterior. Encontramos diferencia estadísticamente significativa de las variables edad de la primera luxación y edad en el momento del estudio en relación con el índice glenohumeral vertical. Por lo tanto, parece lógico que cuanto más joven es el paciente con LRH, mayor es la probabilidad de tener displasia glenohumeral.

No se conoce si otras variables tales como la edad de la primera luxación, la edad en el momento de estudio, el lado afecto, género y la dominancia, pueden tener una implicación con la goniometría en los grupos de estudio. Según nuestros datos no hay relación con esas variables, concordando con la escasa bibliografía. En relación con el lado afecto, Cyprien y $\mathrm{col}^{38}$ comentaba que al comparar los hombros de pacientes con LRH y aquellos de la población normal, no existían diferencias significativas, de acuerdo con el lado en el IGHH. O'Driscoll y Evans ${ }^{52}$, en relación a la aparición de inestabilidad en el hombro contralateral, no encontraban relación con el lado dominante o el género del paciente. A pesar de no mostrar que los parámetros goniométricos varíen con la edad, está demostrado que el pronóstico está influenciado por la edad de la primera luxación, lo cual es evidencia de una cierta predisposición, ya que la luxación en personas jóvenes es un factor predisponente de reci$\operatorname{diva}^{42,53}$, y no así en adultos (menor tasa de recidivas). Friedman y col ${ }^{54}$ no encontraron diferencia en la versión glenoidea respecto al lado, comparando un grupo de hombros inestables y otro grupo control. Krönberg $\mathrm{y} \mathrm{col}^{55}$ confirmaron que no existen diferencias entre hombres y mujeres en lo que respecta a la RH.

\section{Displasia primaria o secundaria}

Descartamos la displasia secundaria en nuestro estudio ya que ninguno de los pacientes y controles estaban relacionados con actividades laborales, deportivas o recreativas de lanzamientos.

Pueden existir alteraciones morfológicas secundarias en deportistas que realizan lanzamientos, sobre todo béisbol. Diversos estudios han demostrado diferencias que lo más probable es que sean 
secundarias a cambios adaptativos, entre hombros de atletas que realizan lanzamientos y los que no lo hacen. Los cambios estructurales en el húmero proximal de pacientes esqueléticamente inmaduros se han evaluado con TAC, documentándose un aumento significativo en la retroversión de la cabeza humeral $(\mathrm{p}<0,001)$ y cavidad glenoidea $(\mathrm{p}<0,01)$ en el lado dominante de 25 lanzadores o pitcher profesionales ${ }^{56}$. Se cree que dichos cambios ocurren durante la inmadurez esquelética en jóvenes pitcher y pueden ser secundarios a estrés repetidos en rotación externa, de acuerdo con la ley de Wolf ${ }^{57}$. Asimismo, la versión humeral y la versión glenoidea medidos por TAC han sido implicados en la etiología del impingement interno y lesiones del manguito rotador $^{58}$.

\section{Inestabilidad en el hombro contralateral}

La aparición de inestabilidad clínica en el hombro sano contralateral es conocida y explicable según los resultados del presente estudio, ya que encuentra afectación displásica también en el hombro sano contralateral tanto en relación al hombro inestable como respecto a la población normal. Esta interpretación no extraña ya que algunos de los factores que contribuyen a la estabilidad glenohumeral están determinados congénitamente $\mathrm{e}^{37,60} \mathrm{y}$, por tanto, es lógico que variaciones individuales de dichos factores puedan tener un efecto en la progresión hacia la inestabilidad de ambos hombros. La incidencia de inestabilidad bilateral en la bibliografía es mayor de lo esperado de forma aleatoria entre los hombros de la población general: Rowe refería un $12 \%$, Moseley y Övergaard $10 \%^{8}$, Morrey y Janes $9 \%$ bilateralmente a la edad de 10 años ${ }^{7}$. Hovelius ${ }^{42}$ encontró $17 \%$ entre $23-29$ años, y buscando una interpretación genética, encontró que el $5 \%$ de otros miembros familiares tenían inestabilidad glenohumeral por $1.7 \%$ de la población general. O'Driscoll y Evans ${ }^{59}$ encontraron una prevalencia de afectación en el hombro contralateral del $14 \%$ a la edad de 5 años, incrementándose hasta el 25\% a los 13 años (con diferencia estadísticamente significativa), afirmando que a mayor tiempo de seguimiento se encuentran mayores tasas de recidivas. La mayor tasa de recidivas en pacientes mayores de 20 años y la afectación del hombro contralateral significativamente era mayor (triple) en aquellos cuya primera luxación ocurrió antes de los 15 años, la achacaban a una anomalía intrínseca, confirmando la existencia de una predisposición congénita bilateral, pero con predominancia unilateral como resultado de diversos factores predisponentes a la inestabilidad. Más tarde Dowdy y O'Driscoll ${ }^{60}$ encontraron que la inestabilidad bilateral era más frecuente en pacientes con historia familiar, siendo inestable el hombro contralateral en el $50 \%$ de los pacientes con historia familiar y del $26 \%$ en aquellos sin ella ( $\mathrm{p}=0,03)$, sugiriendo una predisposición genética. Esos estudios $^{7,8,42,59,60}$ confirman, epidemiológicamente, los resultados de nuestro estudio, concediendo evidencia acerca del componente congénito etiológico en el desencadenamiento y desarrollo de la inestabilidad glenohumeral anterior.

Una vez que la inestabilidad está presente los cambios progresivos en la glenoides, debido a las solicitaciones locales, hacen más inestable a la articulación. Schulz $\mathrm{y} \mathrm{col}^{61}$ realizaron un análisis "in vivo" de la distribución de las fuerzas en la glenoides en pacientes con LRH, por medio de TACabsorciometría. Encontraron que la mineralización del hueso subcondral indicaba una distribución más anterior e inferior de las fuerzas a la que era sometido comparado con los hombros estables. Así, pudieron determinar que en inestabilidades postraumáticas el cambio en la zona anterior era máximo, mientras que en las atraumáticas era principalmente inferior. Por tanto, el cambio de la densidad máxima varía de acuerdo con la causa de la inestabilidad glenohumeral.

Podemos concluir que la etiología de la inestabilidad glenohumeral anterior es multifactorial, pero existe una predisposición anatómica que la favorece y que afec- 
ta, aunque en menor grado, al lado estable contralateral, confirmando el papel de dicha teoría displásica congénita. En el caso de un hombro con dicha predisposición morfológica, la LRH puede desarrollarse como suma de otros factores o bien de un mecanismo traumático que puede causar fractura del reborde antero-inferior o desinserción de labrum y ligamento glenohumeral inferior, lo cual habitualmente cronifica la inestabilidad. Si, además, existe un valor alterado de otros parámetros de displasia, entonces existe una mayor posibilidad de inestabilidad clínica. Su coexistencia con alteraciones en los ligamentos glenohumerales, cápsula articular, músculo subescapular y el manguito rotador, provocará la inestabilidad de la articulación.

Hemos encontrado como parámetros relevantes en la inestabilidad anterior en el estudio realizado: índice glenohumeral horizontal, inclinación glenoidea y ángulo de anteversión de la escápula, siendo discutido el valor de la retroversión humeral. Se precisa de estudios con grandes muestras de pacientes para incrementar el conocimiento del desarrollo de la LRH así como nuevos estudios y valoraciones por otros grupos investigadores para confrontar, confirmar o matizar nuestros resultados.

\section{Agradecimientos}

A la Dra. Eva Ardanaz Aicua por su apoyo metodológico en la realización de este trabajo.

\section{BIBLIOGRAFÍA}

1. Gallino M, Santamaria E, Dor T. Anthropometry of the scapula: clinical and surgical considerations. J Shoulder Elbow Surg 1998; 7: 284-291.

2. Trout TE, Resnick D. Glenoid hipoplasia and its relationship to instability. Skeletal Radiol 1996; 25: 37-40.

3. DAS SP, Roy GS, SAHA AK. Observations of the tilt of the glenoid cavity of scapula. J Anat Soc India 1966; 15: 114.

4. SAHA AK. Dynamic stability of the glenohumeral joint. Acta Orthop Scand 1971; 42: 491505.
5. SAHA AK. Mechanics of elevation of glenohumeral joint. Acta Orthop Scand 1973; 44: 668678.

6. Turkel SJ, Painio MW, Marshall JL, Girgis FG. Stabilizing mechanism preventing anterior dislocation of the glenohumeral joint. J Bone Joint Surg 1981; 63-A: 1208-1217.

7. Morrey BF, JaNES JM. Recurrent anterior dislocation of the shoulder. Long-term follow-up of the Putti-Platt and Bankart procedures. J Bone Joint Surg 1976; 58-A: 252-256.

8. Moseley HF, ÖvergaARd B. The anterior capsular mechanism in recurrent anterior shoulder dislocation of the shoulder: morphological and clinical studies with especial reference to glenoid labrum and gleno-humeral ligament. J Bone Joint Surg 1962; 44-B, 4: 913-927.

9. WiLK KE, ARrigo CA, AndREws JR. Current concepts: the stabilizing structures of the glenohumeral joint. J Orthop Sports Phys Ther 1997; 25: 363-379.

10. Gallie WE. Le mesurier. Recurring dislocations of the shoulder. J Bone Joint Surg 1948; 30-B, 1: 9-18.

11. Merle D’Aubigné R, Tubiana R. Luxation récidivante de l'épaule. Mem Acad Chir (Paris) 1956; 82 (29-30): 930-934.

12. Trillat A, Leclerc-Chalvet F. Luxation récidivante de l'épaule. Ed. Masson, Paris, 1973.

13. García Mata S. Valoración escapulométrica de las inestabilidades recidivantes glenohumerales. Tesis doctoral. Universidad de Navarra, Pamplona (Spain) 1990.

14. Danzig LA, Greenway GD, Resnick D. The HillSachs lesion: An experimental study. Am J Sports Med 1980; 8: 328-332.

15. SAHA AK. Rezidivierende Schulterluxation. Stuttgart, Enke, 1978. En: Saha, 1981.

16. Vasey H, Cyprien JM, Burdet A, Bonvin JC, KRiTSIKIS N, VuAGnat P. La rétrotorsion humérale et la rélation gléno-humérale dans l'épaule normale et les luxations récidivantes. Rev Chir Orthop 1984; Suppl. II, 70: 38-40.

17. KRONBERg M, Broström LA. Humeral head retroversion in patients with unstable humeroscapular joints. Clin Orthop 1990; 260: 207-211.

18. Baratelli M, ParRini M. The unstable shoulder. A CT study. En: Noble J, Galasco CSB. Recent developments in orthopaedic surgery. Manchester University Press 1987; 9: 42-52.

19. Beischer AD, Simmons TD, Torode IP. Glenoid version in children with obstetric brachial plexus palsy. J Pediatr Orthop 1999; 19: 359361 . 
20. García Mata S. Estudio morfológico de la displasia glenohumeral en la luxación posterior recidivante de hombro. Arch Med Dep 1992; 33: 65-77.

21. LAUMANN V, KRAMPS HS. Computed tomography on recurrent shoulder dislocations. From: Balman Y.L., Welsh R.P. Surgery of the shoulder. Philadelphia, Decker, 1984, p. 84.

22. SAHA AK. Recurrent dislocation of the shoulder. George Thieme-Verlag, New 38. York, 1981, 14.

23. Bernageau J, Godefroy D. Le scanner de l'épaule. From: Morvan G, Massare C, Frija G. Le scanner ostéoarticulaire. Téchniques d'utilisation, indications, résultats. Paris, Edit. VIGOT, 1986, 4 171-178.

24. RANDElli M, GAMBrioli PL. Glenohumeral osteometry by computed tomography in normal and unstable shoulders. Clin Orthop 1986, 208: 151-156.

25. Gambrioli PL, Maggi F, Randelli M. Computerized tomography in the investigation of the scapulohumeral instability. Ital J Orthop Traumatol 1985; 11: 223-232.

26. García Mata S, Hidalgo AM, Ibarra P, Martínez Grande M. Precisión de la Tomografía computada en la investigación de la inestabilidad gleno-humeral. Rev Ortop Traum 1989; 33 IB: 384-388.

27. Helweg RH, Nedden D. Double-contrast computed tomographic examination techniques in habitual and recurrent shoulder dislocation. Europ J Radiol 1988; 8: 6-12.

28. Kinnard P, Tricoire JL, Regean-Yues L, Bergeron D. Assessment of the unstable shoulder by computed arthrography. Am J Sports Med 1983; $11: 157$.

29. Pennes DR, Jonsson K, Buckwalter K, Braunstein E, Blasier R, Wojtys E. Computed arthrotomography of the shoulder: comparaison of examinations made with internal and external rotation of the humerus. AJR 1989; 153 : 1017-1019.

30. Rafi M, Firooznia H, Bonamo JJ, Minkoff J, GoLIMBU C. Athlete shoulder injuries: CT arthrographic findings. Radiology 1987; 162: 559564.

31. OZAKI J. Glenohumeral movements of the involuntary inferior and multidirectional instability. Clin Orthop 1989; 238: 107-111.

32. Itoi E, Lee S, Berglund J, Berge L, Kai-Nan An. The effect of a glenoid defect on anteroinferior stability of the shoulder after Bankart repair: a cadaveric study. J Bone Joint Surg 2000; 82-A: 35-45.
33. Yamamoto N, Muraki T, Sperling JW, Steinmann SP, Cofield RH, ItoI E, AN KN. Stabilizing mechanism in bone-grafting of a large glenoid defect. J Bone Joint Surg 2010; 92-A: 20592066.

34. Inui H, Sugamoto K, Miyamoto T, Yoshikawa H, MACHIDA A, Hashimoto J et al. Glenoid shape in atraumatic posterior instability of the shoulder. Clin Orthop 2002; 403: 87-92.

35. Symeonides PP, Hatzokos I, Chistoforides J, PourNARAS J. Humeral head torsion in recurrent anterior dislocation of the shoulder. J Bone Joint Surg 1995; 77-B: 687-690.

36. Wirth MA, Lyons MB, Rockwood CA. Hypoplasia of the glenoid. J Bone Joint Surg 1993; 75A: $1175-1183$

37. Matsen FA, Thomas SC, Rockwood CA. Anterior glenohumeral instability. De: Rockwood Ch.A., Matsen F.A. The Shoulder. WB Saunders Company, Philadelphia, 1990, vol. 1, 14: 526-622.

38. Cyprien JM, Vasey HM, Burdet A, Bonvin JC, KRITSIKIS N, VuAGNAT P. Humeral retrotorsion and glenohumeral relationship in the normal shoulder and in recurrent anterior dislocation (Scapulometry). Clin Orthop 1983; 175: 8-17.

39. MaKi S, Gruen T. Anthropometric study of the glenohumeral joint. Presented in the 22 Annual Meeting of the Orthopaedic Research Society. From Orleans, 1976, January, 28-30. En: Rockwood ChA, Matsen FA. The Shoulder. W.B. Saunders Company, Philadelphia, 1990, vol 1: 227.

40. Kuriyama S, Fujimaki E, Katagiri T, Vemura S. Anterior dislocation of the shoulder joint sustained though skiing. Arthrographic findings and prognosis. Am J Sports Med 1984; 12: 339-346.

41. Brewer BJ, Wubben RC, Carrera GF. Excessive retroversion of the glenoid cavity. A cause of non-traumatic posterior instability of the shoulder. J Bone Joint Surg 1986; 68-A: 724730 .

42. Hoveluus L. Anterior dislocation of the shoulder in teenagers and young adults: five year prognosis. J Bone Joint Surg 1987; 69-A: 393399.

43. Bryce CD, Davison AC, Lewis GS, Wang L, FlemMING DJ, ARmstrong AD. Two-dimensional glenoid version measurements vary with coronal and sagittal scapular rotation. J Bone Joint Surg 2010; 92-A: 692-699.

44. Rouleau DM. Kidder JF, Pons-Villanueva J, Dynamidis S, Defranco M, Walch G. Glenoid ver- 
sion: how to measure it? Validity of different methods in two-dimensional computed tomography scans. J Shoulder Elbow Surg 2010; 19: 1230-1237.

45. Mintzer CM, Waters PM, Brown DJ. Glenoid version in children. J Pediatr Orthp 1996; 16 : 563-566.

46. Schlemmer B, Dosch JC, Gicquel P, Boutemy P, Wolfram R, KempF JF et al. Analyse tomodensitométrique de la rétrotorsion humérale et de la rétroversion glénoidienne. Rev Chir Orthop 2002; 88: 553-560.

47. Barton NJ. Anteversion of the shoulder. A rare clinical sign. En: Bayley I., Kessel L. Shoulder Surgery. Sprinter, Berlin, Heildelberg New York, 1982; 98-100.

48. Debevolse NT, Hyatt GW, Townsend GB. Humeral torsion in recurrent shoulder dislocations. Clin Orthop Relat Res 1971; 76: 87-93.

49. Dunlap K, Shands AR, Hollister LC, Gaul JS, STREIT HA. A new method for determination of torsion of the femur. J Bone Joint Surg 1953; 35-A: 289-311.

50. Kronberg M, Broström LA, SöDERLUnd V. Retroversion of the humeral head in the normal shoulder and its relationship to the normal range of motion. Clin Orthop 1990: 253: 113117.

51. Hernigou P, Duparc E, Hernigou A. Determining humeral retroversion with computed tomography. J Bone Joint Surg 2002; 84-A: 17531762.

52. O'Driscoll SW, Evans DC. The Du Toit staple capsulorrhaphy for recurrent anterior dislocation of the shoulder: twenty years of experience in six Toronto Hospitals. Ameri- can Shoulder and Elbow Surgeons, 4th Open Meeting. Atlanta, 1988. En: (Matsen, 1990).

53. Hovelius L, Erikson K, Fredin H, Hagberg G, HusSENIUS A, Lind B et al. Recurrences after initial dislocation of the shoulder. J Bone Joint Surg 1983; 65-A: 343-349.

54. Friedman RJ, Hawthorne KB, Genez BM. The use of computerized tomography 55 the measurement of glenoid version. J Bone Joint Surg 1992; 74-A: 1032-1037.

55. Kronberg M, Broström LA, SöDERLund V. Normal standards for collum humeri retroversion. Acta Orthop Scand 1988; Suppl. 227, 59: 78.

56. Crockett HC, Gross LB, Wilk KE, Schwartz ML, Reed J, O'Mara J et al. Osseous adaptation and range of motion at the glenohumeral joint in professional baseball pitchers. Am J Sports Med 2002; 30: 20-26.

57. Levine WN, Brandon ML, Stein BS, Gardner TR, Bigliani LU, Ahmad CS. Shoulder adaptive changes in youth baseball players. J Shoulder Elbow Surg 2006; 15: 562-566.

58. Tétreault P, Krueger A, Zuralowski D, Gerber C. Glenoid version and rotator cuff tears. J Orthop Res 2004; 22: 202-207.

59. O'Driscoll SW, EvANS DC. Contralateral shoulder instability following anterior repair. J Bone Joint Surg 1991; 73-B: 941-946.

60. Dowdy PA, O'Driscoll SW. Shoulder Instability. An analysis of family history. J Bone Joint Surg 1993; 75-B: 782-784.

61. Schulz CU, Anetzberger H, Pfahler M, Refior HJ, MÜLLER-GERBL M. Anterior shoulder instability modifies glenoid subchondral bone density. Clin Orthop 2004; 423: 259-263. 